\title{
Spatial updating of map-acquired representation
}

\author{
Chengli Xiao $^{1} \cdot$ Yangmin Lian $^{2} \cdot$ Mary Hegarty $^{3}$
}

Published online: 27 March 2015

(C) Psychonomic Society, Inc. 2015

\begin{abstract}
In the present study, we examined whether people can update a map-acquired spatial representation. The participants learned a spatial layout from a map displayed on a computer screen, and then performed spatial judgments at a novel position either in the same room (Exp. 1) or in a distal room (Exp. 2). They were required to imagine the spatial layout from a perspective aligned with the learning direction, aligned with their facing direction during testing, or toward a novel direction misaligned with the two directions mentioned above. In both the immediate and nonimmediate environments, the participants performed better from the learning direction than from the novel direction, and also performed better from the facing direction than from the novel direction. These results reveal that people establish an orientationspecific spatial representation through map learning, and that they can update a map-acquired representation during locomotion.
\end{abstract}

Keywords Map learning · Spatial updating · Memory alignment effect $\cdot$ Sensorimotor alignment effect

Maps are an effective tool for helping people acquire spatial knowledge and navigate in novel environments. When you plan a journey to a novel city, the first thing you might do is

Chengli Xiao

xiaocl@nju.edu.cn

1 Department of Psychology, School of Social and Behavioral Sciences, Nanjing University, Nanjing 210023, China

2 Institute for International Students, Nanjing University, Nanjing, China

3 Department of Psychological and Brain Sciences, University of California, Santa Barbara, CA, USA read a map of that city. However, your map-acquired spatial knowledge may not guarantee efficient navigation. For example, most maps are oriented north up, so you need to know which direction in the environment is north, to align your map-acquired spatial knowledge to the surroundings in order to infer the direction of a goal location. On the contrary, if someone has acquired spatial knowledge from living in the city for some time, he or she can typically tell the direction to a goal location from any perspective, without first referring to a specific direction (such as north).

These phenomena were investigated in studies in the 1980 s, with the conclusion that spatial representations acquired from maps were orientation-specific, whereas spatial representations derived from direct experience of the environment were orientation-free (Evans \& Pezdek, 1980; Presson, DeLange, \& Hazelrigg, 1989; Presson \& Hazelrigg, 1984; Sholl, 1987). For example, Presson and Hazelrigg had participants learn four-point paths by viewing maps (map condition), by directly viewing the paths (look condition), or by walking along the paths blindfolded (walk condition). Then, participants were blindfolded and wheeled to a location on the path, with their facing directions either aligned or contraligned (i.e., 180 deg different) with the learning perspective, and they were asked to point to other locations on the path. In the look and walk conditions, the participants had equivalent performance from the aligned and contraligned perspectives (absence of an alignment effect). But in the map condition, the participants performed significantly worse when they were contraligned with the learning perspective than when they were aligned with that perspective (an alignment effect). The result was interpreted as showing that spatial representations derived from primary learning (e.g., viewing and/or walking in the immediate environment) and secondary learning (e.g., learned through representations of the environment, such as a map, or language) were different in their orientation 
specificity. Specifically, it was proposed that after primary learning, people established an orientation-free spatial representation that could be equally accessed from any direction, whereas after secondary learning, people established an orientation-specific spatial representation, which was easier to retrieve from the learning direction than from any other direction.

However, later studies indicated that (1)the spatial representation established after primary learning is also orientationspecific (e.g., Mou \& McNamara, 2002; Shelton \& McNamara, 2001) and (2)the absence of an alignment effect in the studies by Presson et al. (1989) might have been due to spatial updating, which enables people to keep track of target objects when they move to the contraligned perspective (Roskos-Ewoldsen, McNamara, Shelton, \& Carr, 1998; Sholl \& Nolin, 1997; Waller, Montello, Richardson, \& Hegarty, 2002). For example, after participants had viewed the same four-point paths that had been used by Presson et al., Waller et al. asked them either to stay in the learning location and keep facing the learning direction (stay condition) or to walk to the to-be-tested location and face the tobe-tested direction (direct walk condition). Participants in the stay condition performed better from the test heading aligned with the learning perspective than from the contraligned perspective, whereas participants in the direct-walk condition performed equivalently from the two perspectives.

Recent studies have further separated orientation-specific memory from the spatial-updating process by dissociating the memory-aligned perspective and the sensorimotor-aligned perspective (Avraamides \& Kelly, 2010; Kelly, Avraamides, \& Loomis, 2007; Mou, McNamara, Valiquette, \& Rump, 2004). In the study by Kelly et al. (2007), after learning a spatial layout, blindfolded participants rotated in place to face a direction different from the learning perspective. Then they performed judgments of direction from three imagined perspectives that were either aligned with the original study view (memory aligned), aligned with their physical facing direction at test (sensorimotor-aligned), or misaligned with both the facing direction at test and the original study view (misaligned). Participants pointed with greater angular errors and longer latencies from the misaligned perspective than from the memory-aligned perspective (a memory alignment effect), suggesting that the participants encoded the spatial layout with a reference direction that was parallel to their viewing direction during learning. Performance from the misaligned perspective was also inferior to that from the sensorimotor-aligned perspective (a sensorimotor alignment effect), suggesting that the participants updated their spatial representation when rotating to the novel facing direction. Kelly et al. proposed that the coexistence of memory and sensorimotor alignment effects was compatible with current theories of spatial cognition (especially with Mou et al., 2004; Sholl, 2001), which suggested that two systems influence people's performance when they locate a target object in the immediate environment: The memory system encodes the orientation-specific spatial memory with a reference direction parallel to the learning perspective, and the updating system codes and updates object locations while an individual moves in that environment.

Although substantial progress has been made in understanding the spatial representations acquired from primary learning, our understanding of map-acquired spatial representations remains limited. If the absence of an alignment effect after primary learning is due to the spatial-updating process, then does the presence of an alignment effect after map learning necessarily mean that people cannot update a mapacquired representation? The answer may be no. In previous studies or map learning (e.g., Presson et al., 1989; Presson \& Hazelrigg, 1984; Richardson, Montello, \& Hegarty, 1999), the alignment effect was tested by comparing the map-aligned perspective to the contraligned perspective. If we analyze these two perspectives with the terms used by Kelly et al. (2007), we find that the aligned perspective is both memory-aligned (participants were aligned with the learning perspective) and sensorimotor-aligned (participants made judgments from a perspective parallel to their facing direction), whereas the contraligned perspective is sensorimotor-aligned (participants made judgments from a perspective parallel to their facing direction) but memory-contraligned (participants faced a direction $180 \mathrm{deg}$ different from the learning perspective). The comparison between aligned and contraligned perspectives in these earlier studies was actually a comparison between memory-aligned and memory-contraligned perspectives, while maintaining a sensorimotor-aligned perspective in both conditions. This suggests two alternative explanations of the observed alignment effect. First, people might have established an orientation-specific representation from the learning perspective, but failed to update it while moving to the contraligned perspective. Second, people might have established and updated an orientation-specific representation, but they might have accumulated error during the updating process while moving to the contraligned perspective.

Moreover, studies of another type of secondary learning have indicated that people can update spatial representations derived from spatial language (Avraamides, Loomis, Klatzky \& Golledge, 2004; Loomis, Lippa, Klatzky, \& Golledge, 2002). Therefore, it seems likely that people can update map-acquired representations as well. In order to test this hypothesis, in the present study we followed the paradigm used by Kelly et al. (2007). After learning a map from one direction, participants faced a different direction and were tested from memory-aligned (a perspective parallel to the learning direction), sensorimotor-aligned (a perspective parallel to participants' facing direction), and misaligned (a perspective parallel to neither of these perspectives) imagined headings. Better (i.e., faster and/or more accurate) performance from 
the memory-aligned perspective than from the misaligned perspective would indicate that people establish an orientationspecific memory during learning. Critically, if people can update a map-acquired spatial representation, they should locate a target object faster and/or more accurately from the sensorimotor-aligned perspective than from the misaligned perspective. In contrast, if people cannot update a mapacquired spatial representation, there should be no difference in the performance from these two perspectives. We also compared performance from the memory-aligned perspective with that from the sensorimotor-aligned perspective. If people located target objects with greater angular errors and/or longer latencies from the sensorimotor-aligned perspective than from the memory-aligned perspective, but performed better and/or faster than from the misaligned perspective, this would suggest that people can update but that error accumulates during the updating process.

In previous research, map learning and testing had been operationalized in different ways, leading to seemingly inconsistent results across studies. For example, in the study by Presson et al. (1989), the participants learned either a large $(4 \times 4 \mathrm{~m})$ or a small $(0.4 \times 0.4 \mathrm{~m})$ map of four-point routes. Then they were blindfolded and guided to a location corresponding to a location on the route just learned, facing a direction that was either aligned or contraligned with the learning direction. If the map was large, the participants performed equivalently in the aligned and contraligned conditions, suggesting that they were able to update their spatial representation. If the map was small, the participants made less-accurate directional judgments (i.e., with more angular error) when their facing direction was contraligned with the learning direction than when it was aligned, suggesting that they were unable to update their spatial representation. In contrast, in one condition of a related study, employing the same type of small four-point-route map $(0.4 \times 0.4 \mathrm{~m})$, Giudice, Betty, and Loomis (2011) had people learn a map and then rotate in place while leaving the map behind them (rotate without map). After rotation, the researchers observed that participants performed equivalently from the learning direction and from their facing direction, suggesting that the participants were able to update their spatial representation.

The discrepancy in the results of these studies can be explained in terms of whether the task required participants to project their map-acquired representation onto a real environment, or whether participants could perform the task by updating the map itself. It is likely that the participants in Giudice et al.'s (2011) study updated the map itself, in contrast with the participants in the small-map condition in Presson et al.'s (1989) study, who had to update an environment corresponding to the small map, and therefore had to project their small-map representation onto that environment. Similarly, the large map in Presson et al.'s study was equivalent to a room-size layout, so participants did not have to project the map-acquired information into the surrounding environment before acting directly on it. Considering that people in daily life usually learn from a small-scale map and then navigate in a large environment corresponding to the map, in the present study we defined spatial updating of a map-acquired representation as spatial updating in the real environment that is represented by the map. We employed the small-scale map paradigm in Presson et al.'s study, in which the participants are guided to a novel position in a real, room-size environment after learning from a small map and are asked to imagine themselves in the larger environment corresponding to the map.

In Experiment 1, participants learned a map of a ten-object array, and then were blindfolded and walked forward to the middle of an open area in front of their study location. They were asked to imagine they were standing at the center of the map and facing parallel to the learning direction. Next, the participants rotated in place to face an object on the map, which had a facing direction with an angular deviation of $240 \mathrm{deg}$ from the learning direction. Then, the participants made spatial judgments from memory-aligned, sensorimotor-aligned, and misaligned perspectives. Experiment 2 was the same as Experiment 1, except that the participants were tested in a distal room. We first describe how the experimental manipulations affected latencies and absolute angular errors in each of the experiments. Then we will combine the data from the two experiments and use circular statistics to decompose the error data into absolute heading errors, pointing variability, and configuration errors, to better understand the factors that contribute to differences in performance from imagined perspectives.

\section{Experiment 1}

\section{Method}

Participants A total of 32 Nanjing University students (16 men and 16 women with normal or corrected-to-normal vision; age range 19-26) participated in this experiment for monetary compensation.

Materials The participants learned a map, shown on a computer screen approximately $40 \mathrm{~cm}$ in front of them. The map had a circular shape $(16.5 \mathrm{~cm}$ in diameter) and depicted a cylindrical space that was $3 \mathrm{~m}$ in diameter. As is shown in Fig. 1, the names of ten objects were surrounded by squares and marked in white. The angles scissors-basket-candle and scissors-basket-ball were $120 \mathrm{deg}$. The map scale was at the bottom right of the screen, and the testing position was $2 \mathrm{~m}$ in front of the learning position, in the middle of a $4 \times 4 \mathrm{~m}$ open area. 


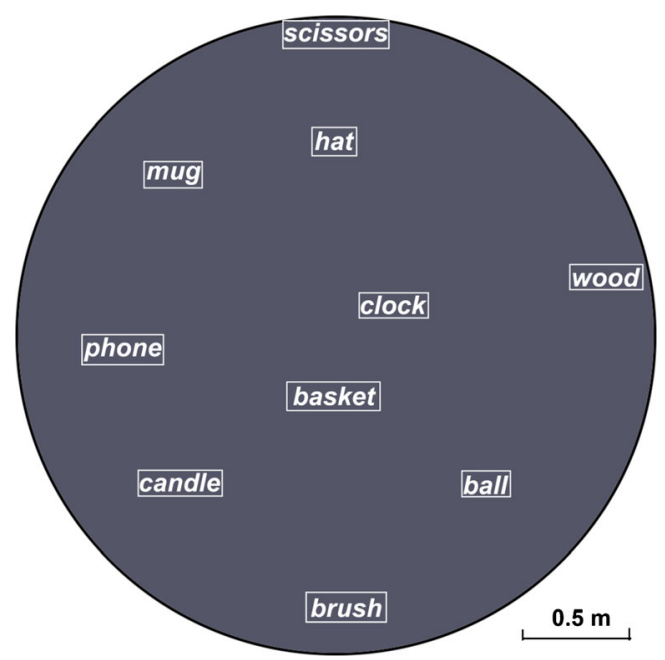

Fig. 1 Map of the object array used in this study

During testing, participants received instructions, including an imagined heading instruction (e.g., "Imagine you are facing the scissors") and a target-pointing instruction (e.g., "Please point to the phone"), via a wireless earphone from the computer. They responded by indicating the direction of the target object with a joystick.

Procedure and design Participants were initially familiarized with the task and the joystick in several practice trials. Then, the experimenter presented a box of the actual objects that would be shown on the map and named each object for the participants. After this, all of the objects were put away so that from then on, the participant only saw maps indicating the locations of the objects.

Participants were then guided to sit in front of a computer and were instructed to study the map on the screen. After a fixation point, participants viewed the map for $30 \mathrm{~s}$ before the presentation of a black-and-white chessboard mask. Participants were then blindfolded and required to name and point to every object on the screen in an arbitrary order. This learning-pointing session was repeated 10 times. All of the participants were able to point object locations quickly and accurately by the 10th time.

After all of the learning-pointing sessions, the participants were blindfolded. The experimenter moved away both the table and the computer that were in front of the participant, and escorted the participant straight forward to the testing position. Then the participants were instructed to imagine themselves in the environment corresponding to the map, standing at the position of the basket and facing the scissors ("Please imagine you are in the environment corresponding to the map, standing at the position of the basket and facing the scissors"). Half of the participants were instructed to turn left (counterclockwise) until they believed they were facing the ball, and the other half to turn right (clockwise) until they believed they were facing the candle (i.e., both groups were asked to turn $240 \mathrm{deg}$ ). After that, all participants completed two blocks of test trials, with each block including 24 trials. Within each block, participants were asked to imagine that they were facing one object (i.e., scissors, candle, or ball) on each trial, and then to point to one of the other eight objects except for the basket and the one they thought they were facing. The three imagined perspectives were aligned with the learning direction (memory-aligned), aligned with their physical facing direction at test (sensorimotor-aligned), or misaligned with their facing direction at test as well as with the original study view (misaligned). For example, a participant who turned to face the ball at test would be asked to imagine that he or she was facing the scissors (memoryaligned perspective), the ball (sensorimotor-aligned perspective), or the candle (misaligned perspective). The trials were presented in a pseudorandom order, such that the imagined facing objects in adjacent trials were not the same. The primary dependent variables were pointing latency (measured as the time from presentation of the name of the target object to the pointing response) and absolute pointing error (measured as the absolute angular difference between the judged pointing direction and the actual direction of the target).

\section{Results and discussion}

The pointing latencies and absolute pointing errors were each subjected to a one-way analysis of variance (ANOVA), with imagined perspective (memory-aligned, sensorimotoraligned, misaligned) as the within-subjects variable.

Pointing latency The main effect of the imagined perspective was significant, $F(2,62)=30.07, p<.001, \eta_{\mathrm{p}}{ }^{2}=.49$. As is shown in Fig. 2, pairwise comparisons showed that the participants performed more quickly when imagining the memoryaligned perspective than when imagining the misaligned perspective (a memory alignment effect), $F(1,31)=38.10, p<$ $.001, \eta_{\mathrm{p}}{ }^{2}=.55,95 \%$ confidence interval (CI) [3.82, 7.59], and more quickly when imagining the sensorimotor-aligned perspective than when imagining the misaligned perspective (a sensorimotor alignment effect), $F(1,31)=16.25, p<.001, \eta_{\mathrm{p}}{ }^{2}$ $=.34$, CI $[1.39,4.25]$. Moreover, the pointing latency was significantly shorter when the participants imagined the memory-aligned perspective rather than the sensorimotoraligned perspective, $F(1,31)=29.75, p<.001, \eta_{\mathrm{p}}{ }^{2}=.49$, CI $[1.81,3.96]$.

Absolute pointing error The main effect of the imagined perspective was significant, $F(2,62)=10.10, p<.001, \eta_{\mathrm{p}}{ }^{2}=$ .25. As is shown in Fig. 3, pairwise comparisons showed that the absolute pointing error was significantly smaller from the memory-aligned perspective than from the misaligned perspective (a memory alignment effect), $F(1,31)=16.26, p<$ 


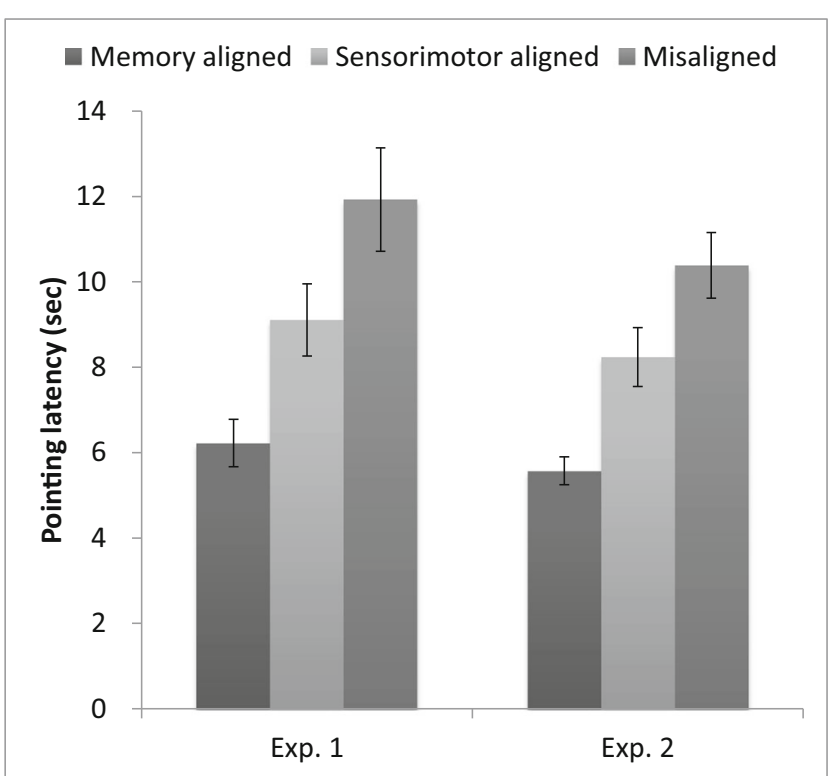

Fig. 2 Pointing latencies in Experiments 1 and 2, as a function of imagined perspective. Error bars are confidence intervals corresponding to $\pm 1 S E M$

$.001, \eta_{\mathrm{p}}{ }^{2}=.34,95 \%$ CI $[5.22,15.90]$; the absolute pointing error was not significantly smaller from the sensorimotoraligned perspective than from the misaligned perspective (i.e., the sensorimotor alignment effect, although in the predicted direction, was not statistically significant), $F(1,31)=$ $2.26, p=.14, \eta_{\mathrm{p}}^{2}=.07$, CI $[-1.23,8.09]$. The absolute pointing error was significantly smaller from the memoryaligned perspective than from the sensorimotor-aligned perspective, $F(1,31)=9.84, p=.004, \eta_{\mathrm{p}}{ }^{2}=.24$, CI $[2.49,11.75]$.

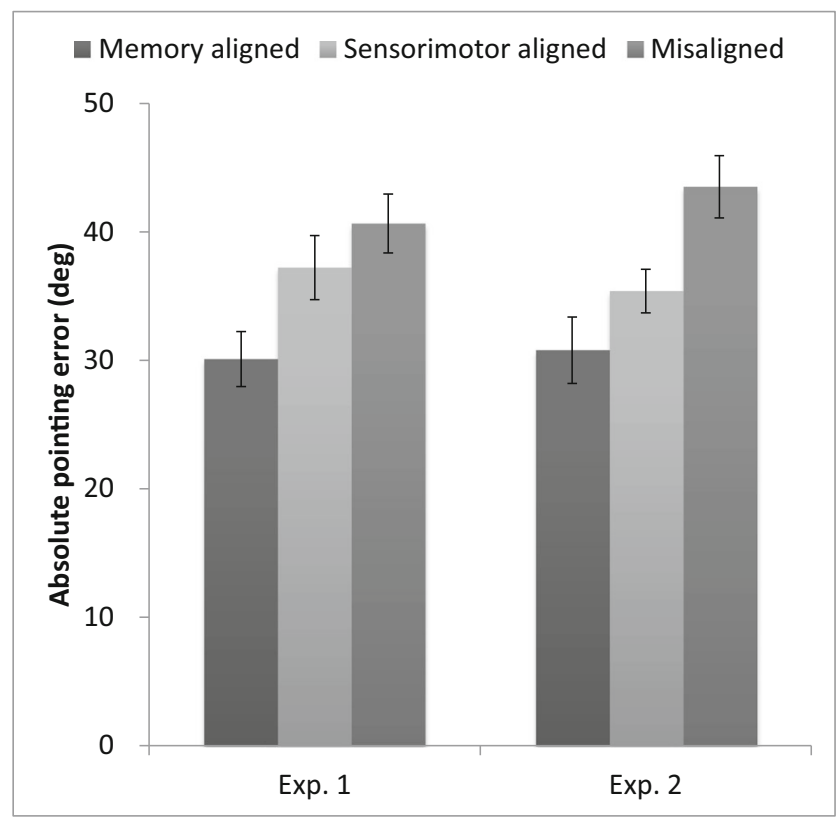

Fig. 3 Absolute pointing errors in Experiments 1 and 2, as a function of imagined perspective. Error bars are confidence intervals corresponding to \pm 1 SEM
In summary, in Experiment 1 the memory alignment effect was consistently found in pointing latencies and absolute pointing errors, suggesting that after learning a map, the participants established a spatial representation with a reference direction that was parallel to the top of the map. The presence of a sensorimotor alignment effect in pointing latencies indicated that the participants also updated the map-acquired representation. There was also a suggestion of a sensorimotor alignment effect in absolute pointing errors, in that errors were also smaller from the sensorimotor-aligned perspective than from the misaligned perspective, but the difference was not significant. A post-hoc power analysis indicated that the power of the test was relatively low (post-hoc power $=.43$; Faul, Erdfelder, Lang, \& Buchner, 2007). Although the sample size of Experiment 1 was larger than those of previous studies with a similar paradigm (Avraamides \& Kelly, 2010; Kelly et al., 2007), the effect size of the sensorimotor alignment effect in absolute pointing errors in Experiment 1 was smaller than those from the previous studies ( $3.43 \mathrm{deg}, d=0.27$, as compared to more than 10 deg in the Avraamides \& Kelly, 2010, study). Although the sensorimotor alignment effect in pointing latencies was clear evidence that participants could update the mapacquired representation, it appears that some error accumulated during the updating process, leading to a small size of the sensorimotor alignment effect in absolute pointing errors.

In Experiment 1, participants were tested in the learning environment, so it was possible that the knowledge of the immediate real environment may have influenced updating of the retrieved map-acquired environment (Waller \& Hodgson, 2006). In Experiment 2, after learning the map, the blindfolded participants were escorted to a novel testing room. We expected that the participants would update the map-acquired environment with less error if they were blind to their immediate environment, so we expected to find a larger effect size in Experiment 2. A power analysis (Faul et al., 2007) indicated that assuming an effect size of 0.4 (i.e., an angular difference between the misaligned and sensorimotor-aligned conditions of approximately $5 \mathrm{deg}$ ), an $N$ of 40 would be needed to obtain statistical power at the recommended .80 level (Cohen, 1988).

In Experiment 1, the absolute pointing error was also larger from the sensorimotor-aligned perspective than from the memory-aligned perspective, and pointing latencies were slower from the sensorimotor-aligned perspective than from the memory-aligned perspective. These results suggest that it takes more time to access the spatial representation in the updating system than that in the memory system.

\section{Experiment 2}

The pointing latency results of Experiment 1 provided preliminary evidence that participants can update an environment 
that that they do not perceive directly (i.e., one that they have learned about only from a map). They might have accomplished this updating process in two possible ways. First, during the learning phase, participants might encode the spatial relations between the objects shown on the map so that they construct an internal representation of the map. At the testing location, they might then project this map-based representation onto the environment that they are situated in and update the projected representation while they are rotating to a novel direction. Second, since an open area was in front of the learning location, participants might have taken advantage of this open area to project the spatial information from the map onto that area during learning, possibly because they guessed the intention of the experiment. In this case, they might have used the spatial cues (such as windows or walls) in the real environment to locate and remember the object locations. A previous study had shown that this strategy is effective for updating described scenes (Avraamides \& Kelly, 2010).

In order to differentiate these two possibilities, in Experiment 2 the participants first learned the map in a crowded faculty office with no open area and then were led to a distal room for testing. If the spatial updating in Experiment 1 was due to the deliberate combination of map knowledge and the open area, the sensorimotor alignment effect should be absent when participants were tested in the distal room. On the contrary, if people can update a mapacquired representation without combining it with the surrounding environment during learning, the sensorimotor alignment effect should occur even in a room that is distal from the learning environment.

\section{Method}

Participants A total of 40 Nanjing University students (20 men and 20 women with normal or corrected-to-normal vision), ranging in age from 19 to 26 years $(M=21.83, S D=$ $2.15)$, participated in this experiment for monetary compensation.

Materials, design, and procedure The experiment was conducted in the same way as Experiment 1, except that the participants learned the map in a furnished faculty office. The faculty office measured $3 \times 4 \mathrm{~m}$, was furnished with typical office furniture (e.g., bookshelves, sofa, desks, and chairs), and included no open area. After learning the map, the participants were blindfolded and guided on a meandering route to the distal testing room, which was the same as the learning/ testing room in Experiment 1. Standing at the testing position, they were asked to imagine themselves in the environment corresponding to the map, standing at the basket and facing the scissors. After that, they were asked to turn left/right to face the ball/candle and performed the same spatial task as in Experiment 1.

\section{Results and discussion}

The pointing latencies and absolute pointing errors were subjected to a one-way ANOVA with imagined perspective (memory-aligned, sensorimotor-aligned, misaligned) as the within-subjects variable.

Pointing latency The main effect of the imagined perspective was significant, $F(2,78)=42.50, p<.001, \eta_{\mathrm{p}}{ }^{2}=.52$. As is shown in Fig. 2, pairwise comparisons showed that the participants performed more quickly when imagining the memoryaligned perspective than when imagining the misaligned perspective (a memory alignment effect), $F(1,39)=71.41, p$ $<.001, \eta_{\mathrm{p}}{ }^{2}=.65,95 \% \mathrm{CI}[3.66,5.97]$, and more quickly when imagining the sensorimotor-aligned perspective than when imagining the misaligned perspective (a sensorimotor alignment effect), $F(1,39)=27.62, p<.001, \eta_{\mathrm{p}}{ }^{2}=.42$, CI [1.32, 2.97]. Moreover, the pointing latency was significantly smaller from the memory-aligned perspective than from the sensorimotor-aligned perspective, $F(1,39)=21.57, p<$ $.001, \eta_{\mathrm{p}}{ }^{2}=.36$, CI [1.51, 3.83].

Absolute pointing error The main effect of imagined perspective was significant, $F(2,78)=12.74, p<.001, \eta_{\mathrm{p}}{ }^{2}=$ .25. As is shown in Fig. 3, pairwise comparisons showed that the absolute pointing error was significantly smaller when the participants imagined the memory-aligned rather than the misaligned perspective (a memory alignment effect), $F(1$, $39)=23.79, p<.001, \eta_{\mathrm{p}}{ }^{2}=.38,95 \%$ CI $[7.45,18.00]$, and the absolute pointing error was significantly smaller when they imagined the sensorimotor-aligned rather than the misaligned perspective (a sensorimotor alignment effect), $F(1,39)=11.78, p=.001, \eta_{\mathrm{p}}{ }^{2}=.23$, CI $[3.33,12.91]$. The absolute pointing error was marginally significantly smaller when the participants imagined the memory-aligned perspective than when they imagined the sensorimotor-aligned perspective, $F(1,39)=2.97, p=.09, \eta_{\mathrm{p}}{ }^{2}=.07, \mathrm{CI}[-0.80,10.01]$.

The results of Experiment 2 were similar to those of Experiment 1: Participants performed better (faster and with less absolute pointing error) from the memoryaligned perspective than from the misaligned perspective; they also responded faster from the sensorimotor-aligned than from the misaligned perspective. Critically, the sensorimotor alignment effect was also evident in absolute pointing errors in this experiment (post hoc power $=.96$ ). These results indicate that participants could retrieve the map-acquired representation and project and update it in a novel environment that they had never perceived. Since there was no open area in the faculty office, it is unlikely that the participants deliberately projected the map representation and combined it with the surrounding environment during learning. Moreover, participants performed the spatial task on the basis of memory alone, because they 
were blindfolded before entering the testing room and throughout the testing phase.

Interestingly, as compared with Experiment 1, the angular difference between the misaligned and sensorimotor-aligned headings in Experiment 2 increased to $8.12 \mathrm{deg}(d=0.54)$, supporting our hypothesis that the novelty of the immediate environment was beneficial for participants in updating the retrieved map-acquired representation. The difference between the memory-aligned and sensorimotor-aligned perspectives was only marginally significant for this measure, also supporting this hypothesis.

Because the only difference between Experiments 1 and 2 was the testing location, the absolute pointing error data from the two experiments were combined and subjected to a mixed-model ANOVA, with imagined perspective (memory-aligned, sensorimotor-aligned, misaligned) as the within-subjects variable and testing location (same room, distal room) as the between-subjects variable. The main effect of imagined perspective was significant, $F(2,140)$ $=21.27, p<.001, \eta_{\mathrm{p}}{ }^{2}=.23$. Pairwise comparisons showed that the absolute pointing error from the memory-aligned perspective was significantly smaller than that from the misaligned perspective (a memory alignment effect), $F(1$, $70)=38.73, p<.001, \eta_{\mathrm{p}}{ }^{2}=.36,95 \% \mathrm{CI}[7.91,15.37]$, and smaller than that from the sensorimotor-aligned perspective, $F(1,70)=10.53, p=.002, \eta_{\mathrm{p}}{ }^{2}=.13$, CI $[2.26,9.47]$. The absolute pointing error was also significantly smaller from the sensorimotor-aligned perspective than from the misaligned perspective (a sensorimotor alignment effect), $F(1,70)=11.94, p=.001, \eta_{\mathrm{p}}{ }^{2}=.15$, CI $[2.44,9.11]$. Neither the main effect of testing location nor the interaction between testing location and imagined perspective was significant, $F \mathbf{s}<1$.

\section{Analysis of sources of pointing errors}

The absolute pointing error indicated that people performed differently from different imagined perspectives. To provide further insight into the factors that cause these differences, we calculated the signed pointing errors for each trial. Then, following the analysis method introduced by Wang and Spelke (2000) and adapted by Mou, McNamara, Rump, and Xiao (2006; see also Xiao, Mou, \& McNamara, 2009; Xiao \& Zhang, 2013), we computed each participant's heading error, pointing variability, and configuration error from each imagined perspective. This type of analysis of pointing errors had not been conducted in the previous studies of map learning discussed earlier (e.g., Giudice et al., 2011; Presson et al., 1989; Presson \& Hazelrigg, 1984), and so is novel to this study.

Heading error is defined as the mean of all the signed pointing errors across trials, and this was computed for each participant from each imagined perspective. This measures constant bias in pointing; in ideal performance, the heading error would be 0 . Because there was no consistent direction of bias across participants, we took the absolute value of each participant's heading error and averaged this across participants to compute the absolute heading error, to measure the extent of the constant bias across participants. Pointing variability is defined as the square root of the mean of the variance of the signed errors for each target object, and it measures consistency in how people point to the same object over trials; more variance indicates more uncertainty about the location of the objects. Configuration error is defined as the standard deviation of the means of the signed pointing errors per target object, which measures the fidelity of the object-toobject spatial relations. If participants located objects very accurately, the signed pointing error per target object would be 0 , and the configuration error would also be 0 . If participants had a consistent bias when retrieving their spatial representations but preserved the object-to-object spatial relations very accurately, their pointing error per target object would increase by the same amount in the same direction for each object. In this situation, the configuration error would remain 0 . The configuration error only increases when the fidelity of object-to-object spatial relations is lower.

Because the only difference between Experiments 1 and 2 was the testing location, and because of the possible low power in Experiment 1, the measures of absolute heading error, pointing variability, and configuration error in Experiments 1 and 2 were combined and subjected to mixed-model ANOVAs, with imagined perspective (memory-aligned, sensorimotor-aligned, misaligned) as the within-subjects variable and testing location (same room, distal room) as the betweensubjects variable. The main effect of imagined perspective was significant for absolute heading errors, $F(2,140)=$ 26.59, $p<.001, \eta_{\mathrm{p}}{ }^{2}=.28$, and pointing variability, $F(2,140)$ $=13.53, p<.001, \eta_{\mathrm{p}}{ }^{2}=.16$. As is shown in Table 1, pairwise comparisons indicated that the absolute heading error was smaller from the memory-aligned perspective than from both the misaligned perspective, $F(1,70)=54.25, p<.001, \eta_{\mathrm{p}}{ }^{2}=$ $.44,95 \% \mathrm{CI}[11.28,19.65]$, and the sensorimotor-aligned perspective, $F(1,70)=32.05, p<.001, \eta_{\mathrm{p}}{ }^{2}=.31$, CI [7.01, 14.63]. The absolute heading error was marginally smaller from the sensorimotor-aligned perspective than from the misaligned perspective, $F(1,70)=3.51, p=.07, \eta_{\mathrm{p}}{ }^{2}=.05$, CI $[-0.30,9.59]$. For pointing variability, the differences among the three imagined perspectives were all significant. The pointing variability was smaller from the memoryaligned perspective than from both the misaligned perspective, $F(1,70)=26.36, p<.001, \eta_{\mathrm{p}}{ }^{2}=.27$, CI [8.26, 18.74], and the sensorimotor-aligned perspective, $F(1,70)=6.77, p=$ $.01, \eta_{\mathrm{p}}{ }^{2}=.09, \mathrm{CI}[1.62,12.25]$. The pointing variability was also smaller from the sensorimotor-aligned perspective than from the misaligned perspective, $F(1,70)=6.96, p=.01, \eta_{\mathrm{p}}{ }^{2}$ 
Table 1 Means (and standard deviations) of absolute heading errors, pointing variability, and configuration errors, as a function of imagined perspective

\begin{tabular}{lllr}
\hline & \multicolumn{2}{l}{ Imagined Perspective } & \\
\cline { 2 - 4 } & Memory Aligned & Sensorimotor Aligned & Misaligned \\
\hline Absolute heading error & $7.20(6.29)$ & $18.06(15.10)$ & $22.97(17.75)$ \\
Pointing variability & $25.70(16.88)$ & $32.44(16.40)$ & $39.32(16.88)$ \\
Configuration error & $40.01(21.27)$ & $34.89(15.74)$ & $38.30(14.80)$ \\
\hline
\end{tabular}

$=.09, \mathrm{CI}[1.60,11.53]$. However, there were no significant differences among the three imagined perspectives in configuration errors, $F(2,140)=2.19, p=.12, \eta_{\mathrm{p}}{ }^{2}=.03$. Finally, the main effect of testing location was significant for pointing variability, $F(1,70)=6.42, p=.013, \eta_{\mathrm{p}}{ }^{2}=.08$; participants who were tested in the learning room pointed to target objects with more variance than did participants tested in the distal room. No other effects were significant.

In summary, the analysis of sources of error revealed that the memory alignment effect was evident in both absolute heading errors and pointing variability, and the sensorimotor alignment effect was evident primarily in pointing variability, with a marginal effect for absolute heading errors. The difference between the memory-aligned perspective and the sensorimotor-aligned perspective was also evident in both absolute heading errors and pointing variability. However, we found no evidence of differences in configuration errors for the three imagined perspectives. The testing location only affected pointing variability: Participants pointed with greater variability when tested in the learning room than when in the distal room.

\section{General discussion}

In two experiments, participants learned the layout of a spatial array from a map, and we compared pointing performance from three different imagined perspectives: one that was aligned with the participants' perspective during learning (memory-aligned), one that was aligned with their body orientation during testing (sensorimotor-aligned), and a novel perspective that was aligned with neither of these (misaligned). This design contrasts with those in previous studies of map-acquired spatial representations, which tested only memory-aligned and -contraligned perspectives (e.g., Presson \& Hazelrigg, 1984). The superior performance for the memory-aligned perspective in our study indicated that participants established a spatial memory with a reference direction, which was based on the orientation of the map during learning. The superior performance for the sensorimotoraligned perspective relative to the misaligned perspective indicated that participants could update their map-acquired spatial memory during rotation. Furthermore, they performed better from the memory-aligned perspective than from the sensorimotor-aligned perspective, suggesting that although they could update the map-acquired representation, some error accumulated during movement, and further analyses indicated that this accumulated error included both constant bias and uncertainty, but did not affect the fidelity of object-to-object spatial relations.

These results are consistent with the presence of alignment effects in previous studies of map-acquired representations (e.g., Presson et al., 1989; Presson \& Hazelrigg, 1984; Richardson et al., 1999; Sholl, 1987). In the introduction, we raised two alternative possible explanations of the alignment effects in these previous studies. One explanation was that people establish an orientation-specific representation but fail to update it during rotation; the other was that people can update the orientation-specific representation but accumulate error during rotation. The present results favor the latter explanation, in that we found evidence for better performance (fewer errors and shorter latencies) for sensorimotor-aligned than for misaligned perspectives.

However, we should note that the present study does not exclude the possibility that participants failed to update in the earlier studies of map-acquired representations (i.e., Presson et al., 1989; Presson \& Hazelrigg, 1984; Richardson et al., 1999; Sholl, 1987). Most current theories of spatial cognition agree on the dichotomy of an online spatial-updating system and an offline spatial memory system (Avraamides \& Kelly, 2008; Burgess, 2006; Mou, Fan, McNamara, \& Owen, 2008; Sholl, 2001; Waller \& Hodgson, 2006; Wang \& Spelke, 2002). In most circumstances, the spatial-updating system keeps track of the individual's immediate real environment (online spatial representation) but not remote and/or imagined environments (offline spatial memories). Only under certain conditions, which encourage people to connect the spatialupdating system and the retrieved spatial memory, are people able to update the remote and/or imagined environment (Avraamides, Galati, \& Papadopoulou, 2013; Avraamides \& Kelly, 2008; Kelly et al., 2007). One such condition may be when people are asked to imagine that they are in a distal environment surrounded by target objects. In the present study, participants were given instructions to imagine that they 
were in the environment they learned from the map. However, in previous studies of map-based representations (e.g., Presson et al., 1989; Presson \& Hazelrigg, 1984; Richardson et al., 1999; Sholl, 1987), there were no such instructions. It is possible that although people can update a map-based representation, they did not spontaneously project their map-based representations to the surrounding environments in previous studies of map-acquired representations; that is, the instructions in these studies did not create the conditions under which people can update their map-based representations.

For example, in the study by Presson and Hazelrigg (1984), after participants had learned the map, no instruction guided them to deliberately imagine that they were in the environment corresponding to the map. The participants were blindfolded and wheeled to the testing location, facing the opposite direction from the learning perspective. Then they were informed that they were in a particular location and facing direction on the route and were asked to point to an object. It is highly likely that under these circumstances, their mapacquired representation did not connect with their online updating system. That is, when wheeled from the learning position to the testing location, it is likely that the participants only updated their immediate environment, and not the mapacquired environment, and then after being wheeled, they retrieved their map-acquired representation in offline memory from the learning perspective during testing.

The analysis of sources of pointing error indicated that the accumulated errors during spatial updating included constant bias (heading error) and the uncertainty of the representation (pointing variability), but not the fidelity of object-to-object spatial relations (configuration error). According to Kelly et al. (2007), the presence of a memory alignment effect and a sensorimotor alignment effect indicate the coexistence of spatial representations in the memory system and the updating system. The present results suggest that the representation in the memory system is the most accurate (i.e., has the least consistent bias and uncertainty). When rotating to a novel direction, it appears that although people do update object locations, they accumulate both consistent bias and uncertainty of the representation. During testing, when people were asked to imagine the array from the misaligned perspective, they had to either mentally rotate the array with respect to themselves or mentally rotate themselves with respect to the array. This mental rotation process also increased both the constant bias and the uncertainty of the representation.

It is interesting that people also took longer to locate targets from the sensorimotor-aligned perspective than from the memory-aligned perspective. There are two possible explanations of this result. One is that the latency might reflect the additional uncertainty of the representation following updating, because it takes longer to decide on a response based on an uncertain representation. Another possibility is that access to spatial representations in memory is faster than access to spatial representations in the updating system. In the present study, people had to switch among three imagined perspectives from trial to trial, which might have led to interference between the retrieved, updated, and imagined representations (cf. May, 2004).

Neither the spatial-updating nor the mental rotation processes affected the fidelity of the object-to-object spatial relations. It is perhaps not surprising that the object-to-object spatial relations remained the same after spatial updating and mental rotation, because a previous study had indicated that after learning object-to-object relations from a map, these relations are preserved even after participants are disoriented (Xiao \& Zhang, 2013).

Another notable finding is that participants who were tested in the learning room pointed to target objects with more variance than did participants tested in the novel room (apart from pointing variability, the two groups performed equivalently in all other dependent variables). This difference might be attributed to interference from the surrounding real environment (Waller \& Hodgson, 2006). When participants were tested in the environment that they had seen during learning (in Exp. 1), they had to project their map-acquired representation to an imagined environment that overlapped with their current surrounding real environment. During updating, they probably automatically updated their surrounding real environment (Wang, 2004), which might have interfered with updating the projected imagined map-acquired representation, reducing the precision of their imagined environment. In Experiment 2, however, the participants had no knowledge of the surrounding environment during testing because they were blindfolded before entering the testing room, thus minimizing any interference from the immediate environment. It is interesting that knowledge of the immediate environment only affected the precision (pointing variability), but not the accuracy (heading or configuration error), of the projected imagined mapacquired representation, suggesting that knowledge of the immediate environment interfered with the imagined environment, adding variance to the estimates of object locations.

In this study, we chose to study map updating in a vistascale environment (cf. Montello, 1993) - that is, an environment (in this case a room) that is visible from a single perspective. In everyday life, maps are more typically used for navigating to unseen locations in environmental-scale spaces, such as a city or building, that can only be apprehended by moving through the environment. Our first reason for studying map updating in a vista-scale environment was for consistency with previous research (Evans \& Pezdek, 1980; Presson et al., 1989; Presson \& Hazelrigg, 1984; Sholl, 1987). A second reason was that we wished to isolate the process of map updating from other aspects of navigating, such as planning and executing the best route to a goal location. A third reason was that map updating in a room-scale environment is an ecologically valid task, in that people often use maps in 
environments of this scale - for example, in planning the rearrangement of furniture in a room. In future studies, it will be important to study how people update map-based representations when navigating unseen distal target location(s).

In summary, in this study we found that after learning a map, people established an orientation-dependent memory, and that they could project this memory into an imagined environment and update it during movement. As compared to imagining facing a novel perspective, people's physical movements helped them keep track of their current heading and maintain a relatively precise representation of the spatial array. This spatial-updating process is effortful and introduces errors-specifically, heading error and pointing variability. However, the object-to-object spatial relations are preserved during the spatial-updating and mental rotation processes, indicating that contrary to earlier accounts, people can update map-acquired representations.

Acknowledgments We thank Pei Wu for assistance in the data collection, and Margaret Tarampi for discussions and comments on the manuscript. Preparation of this article and the research reported in it were supported by a grant from the National Natural Science Foundation of China, Grant No. 31000457 to C.X.

\section{Reference}

Avraamides, M. N., Galati, A., \& Papadopoulou, C. (2013). Egocentric updating of remote locations. Psychological Research, 77, 716-727. doi:10.1007/s00426-012-0465-5

Avraamides, M. N., \& Kelly, J. W. (2008). Multiple systems of spatial memory and action. Cognitive Processing, 9, 93-106. doi:10.1007/ s10339-007-0188-5

Avraamides, M. N., \& Kelly, J. W. (2010). Multiple systems of spatial memory: Evidence from described scenes. Journal of Experimental Psychology: Learning, Memory, and Cognition, 36, 635-645. doi: 10.1037/a0017040

Avraamides, M. N., Loomis, J. M., Klatzky, R. L., \& Golledge, R. G. (2004). Functional equivalence of spatial representations derived from vision and language: Evidence from allocentric judgments. Journal of Experimental Psychology: Learning, Memory, and Cognition, 30, 801-814. doi:10.1037/0278-7393.30.4.801

Burgess, N. (2006). Spatial memory: How egocentric and allocentric combine. Trends in Cognitive Sciences, 10, 551-557. doi:10.1016/ j.tics.2006.10.005

Cohen, J. (1988). Statistical power analysis for the behavioral sciences (2nd ed.). Hillsdale, NJ: Erlbaum.

Evans, G. W., \& Pezdek, K. (1980). Cognitive mapping: Knowledge of real-world distance and location information. Journal of Experimental Psychology: Human Learning and Memory, 6, 1324. doi:10.1037/0278-7393.6.1.13

Faul, F., Erdfelder, E., Lang, A.-G., \& Buchner, A. (2007). G*Power 3: A flexible statistical power analysis program for the social, behavioral, and biomedical sciences. Behavior Research Methods, 39, 175-191. doi:10.3758/BF03193146

Giudice, N. A., Betty, M. R., \& Loomis, J. M. (2011). Functional equivalence of spatial images from touch and vision: Evidence from spatial updating in blind and sighted individuals. Journal of Experimental Psychology: Learning, Memory, and Cognition, 37, 621-634. doi:10.1037/a0022331
Kelly, J. W., Avraamides, M. N., \& Loomis, J. M. (2007). Sensorimotor alignment effects in the learning environment and in novel environments. Journal of Experimental Psychology: Learning, Memory, and Cognition, 33, 1092-1107. doi:10.1037/0278-7393.33.6.1092

Loomis, J. M., Lippa, Y., Klatzky, R. L., \& Golledge, R. G. (2002). Spatial updating of locations specified by 3-D sound and spatial language. Journal of Experimental Psychology: Learning, Memory, and Cognition, 28, 335-345. doi:10.1037/0278-7393.28.2.335

May, M. (2004). Imaginal perspective switches in remembered environments: Transformation versus interference accounts. Cognitive Psychology, 48, 163-206. doi:10.1016/S0010-0285(03)00127-0

Montello, D. R. (1993). Scale and multiple psychologies of space. In A. U. Frank \& I. Campari (Eds.), Spatial information theory: A theoretical basis for GIS (pp. 312-321). Berlin, Germany: Springer.

Mou, W., Fan, Y., McNamara, T. P., \& Owen, C. B. (2008). Intrinsic frames of reference and egocentric viewpoints in scene recognition. Cognition, 106, 750-769. doi:10.1016/j.cognition.2007.04.009

Mou, W., \& McNamara, T. P. (2002). Intrinsic frames of reference in spatial memory. Journal of Experimental Psychology: Learning, Memory, and Cognition, 28, 162-170. doi:10.1037/0278-7393.28. 1.162

Mou, W., McNamara, T. P., Rump, B., \& Xiao, C. (2006). Roles of egocentric and allocentric spatial representations in locomotion and reorientation. Journal of Experimental Psychology: Learning, Memory, and Cognition, 32, 1274-1290. doi:10.1037/0278-7393. 32.6.1274

Mou, W., McNamara, T. P., Valiquette, C. M., \& Rump, B. (2004). Allocentric and egocentric updating of spatial memories. Journal of Experimental Psychology: Learning, Memory, and Cognition, 30, 142-157. doi:10.1037/0278-7393.30.1.142

Presson, C. C., DeLange, N., \& Hazelrigg, M. D. (1989). Orientation specificity in spatial memory: What makes a path different from a map of the path? Journal of Experimental Psychology: Learning, Memory, and Cognition, 15, 887-897. doi:10.1037/0278-7393.15. 5.887

Presson, C. C., \& Hazelrigg, M. D. (1984). Building spatial representations through primary and secondary learning. Journal of Experimental Psychology: Learning, Memory, and Cognition, 10, 716-722. doi:10.1037/0278-7393.10.4.716

Richardson, A. E., Montello, D. R., \& Hegarty, M. (1999). Spatial knowledge acquisition from maps and from navigation in real and virtual environments. Memory \& Cognition, 27, 741-750. doi:10.3758/ BF03211566

Roskos-Ewoldsen, B., McNamara, T. P., Shelton, A. L., \& Carr, W. (1998). Mental representations of large and small spatial layouts are orientation dependent. Journal of Experimental Psychology: Learning, Memory, and Cognition, 24, 215-226. doi:10.1037/ 0278-7393.24.1.215

Shelton, A. L., \& McNamara, T. P. (2001). Systems of spatial reference in human memory. Cognitive Psychology, 43, 274-310. doi:10.1006/ cogp. 2001.0758

Sholl, M. J. (1987). Cognitive maps as orienting schemata. Journal of Experimental Psychology: Learning, Memory, and Cognition, 13, 615-628. doi:10.1037/0278-7393.13.4.615

Sholl, M. J. (2001). The role of a self-reference system in spatial navigation. In D. R. Montello (Ed.), Spatial information theory: Foundations of geographic information science (pp. 217-232). Berlin, Germany: Springer.

Sholl, M. J., \& Nolin, T. L. (1997). Orientation specificity in representations of place. Journal of Experimental Psychology: Learning, Memory, and Cognition, 23, 1494-1507. doi:10.1037/0278-7393. 23.6.1494

Waller, D., \& Hodgson, E. (2006). Transient and enduring spatial representations under disorientation and self-rotation. Journal of Experimental Psychology: Learning, Memory, and Cognition, 32, 867-882. doi:10.1037/0278-7393.32.4.867 
Waller, D., Montello, D. R., Richardson, A. E., \& Hegarty, M. (2002). Orientation specificity and spatial updating of memories for layouts. Journal of Experimental Psychology: Learning, Memory, and Cognition, 28, 1051-1063. doi:10.1037/0278-7393.28.6.1051

Wang, R. F. (2004). Between reality and imagination: When is spatial updating automatic? Perception \& Psychophysics, 66, 68-76. doi: 10.3758/BF03194862

Wang, R. F., \& Spelke, E. S. (2000). Updating egocentric representations in human navigation. Cognition, 77, 215-250. doi:10.1016/S00100277(00)00105-0
Wang, R. F., \& Spelke, E. S. (2002). Human spatial representation: Insights from animals. Trends in Cognitive Sciences, 6, 376-382. doi:10.1016/S1364-6613(02)01961-7

Xiao, C., Mou, W., \& McNamara, T. P. (2009). Use of self-to-object and object-to-object spatial relations in locomotion. Journal of Experimental Psychology: Learning, Memory, and Cognition, 35, 1137-1147. doi:10.1037/a0016273

Xiao, C., \& Zhang, L. (2013). Egocentric representation acquired from offline map learning. PLoS ONE, 8, e60194. doi:10.1371/journal. pone. 0060194 